Provided for non-commercial research and education use. Not for reproduction, distribution or commercial use.

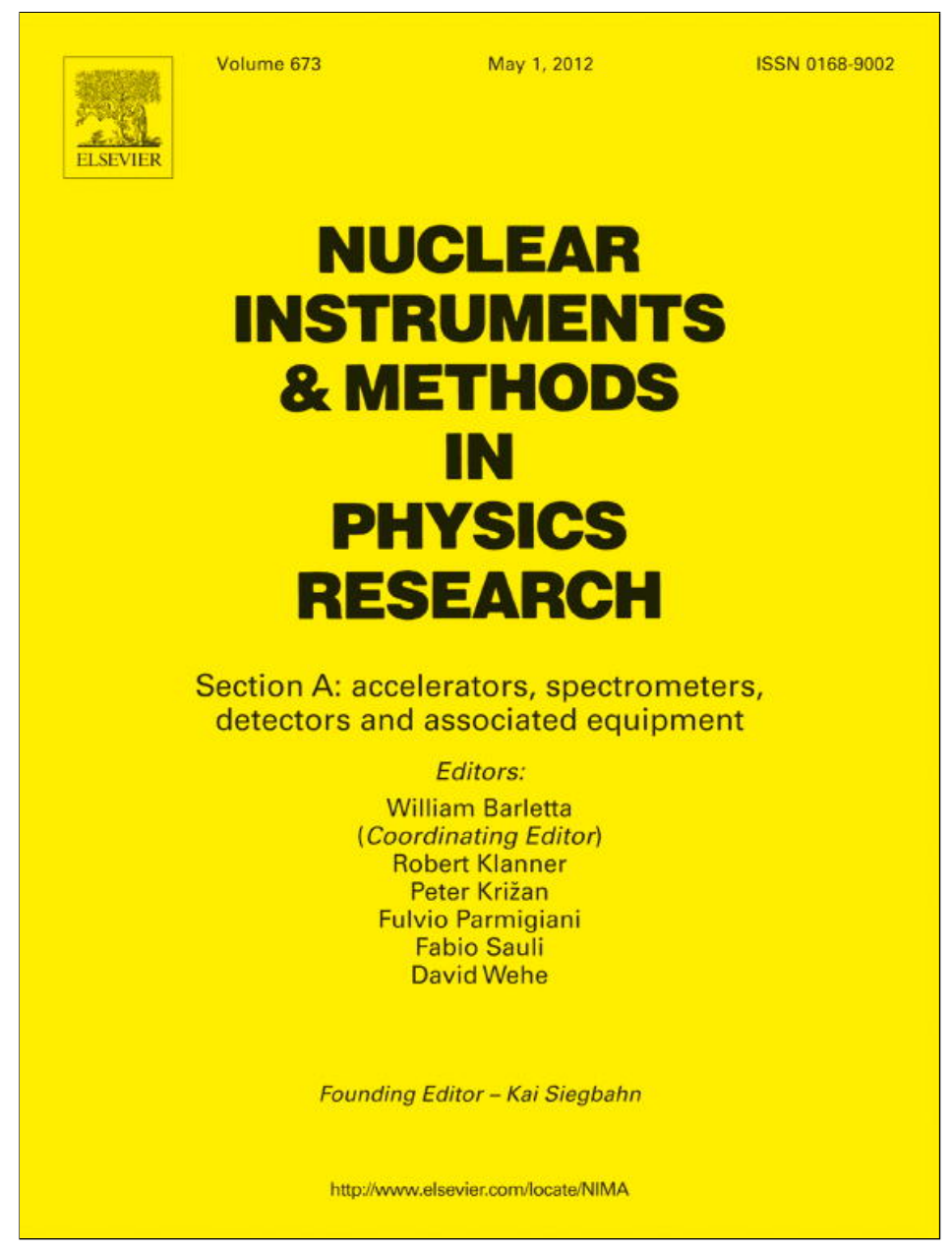

This article appeared in a journal published by Elsevier. The attached copy is furnished to the author for internal non-commercial research and education use, including for instruction at the authors institution and sharing with colleagues.

Other uses, including reproduction and distribution, or selling or licensing copies, or posting to personal, institutional or third party websites are prohibited.

In most cases authors are permitted to post their version of the article (e.g. in Word or Tex form) to their personal website or institutional repository. Authors requiring further information regarding Elsevier's archiving and manuscript policies are encouraged to visit:

http://www.elsevier.com/copyright 


\title{
Enhancing the performances of a resonance detector spectrometer for deep inelastic neutron scattering measurements
}

\author{
A. Filabozzi ${ }^{\mathrm{a}, *}$, E. Pace ${ }^{\mathrm{a}}$, A. Pietropaolo ${ }^{\mathrm{b}}$ \\ a Univ. degli Studi di Roma Tor Vergata and INFN Sez. di Roma Tor Vergata, Italy \\ ${ }^{\mathrm{b}}$ CNISM, UdR di Roma Tor Vergata, Italy
}

\section{A R T I C L E I N F O}

\section{Article history:}

Received 24 June 2011

Received in revised form

23 November 2011

Accepted 27 December 2011

Available online 12 January 2012

Keywords:

Resonance detector

Neutron instrumentation

Gamma detectors

\begin{abstract}
A B S T R A C T
The possibility is explored to sum up neutron Compton profiles at different scattering angles in deep inelastic neutron scattering measurements within the Resonance Detector (RD) configuration to enhance the statistics for a more reliable extraction of the momentum distribution of the constituents in the target. The RD configuration allows to select the energy of the scattered neutrons up to several tens of electron Volt, thus accessing energy and wave vector transfers well above $1 \mathrm{eV}$ and $30 \AA^{-1}$, respectively. In the high- $q / \omega$ regime, the final state effects could be considered as negligible, as shown in a series of simulations using a Monte Carlo method with different inverse geometry instrument setups. The simulations show that it could be possible to conceive an instrument set up where the RD configuration allows the proper summation of several spectra at different scattering angles, providing a good separation of the proton recoil signal from that of the heavier atoms, thus avoiding the cell subtraction by fitting procedure.
\end{abstract}

(c) 2012 Elsevier B.V. All rights reserved.

\section{Introduction}

Among the different experimental techniques relying on neutron probes for the investigation of condensed matter, the Deep Inelastic Neutron Scattering $[1,2]$ is unique in providing information on the short time (sub-femtosecond) microscopic dynamics of hydrogencontaining molecular systems [3-7], as well as of quantum fluids and solids $[8,9]$ and on the corresponding effective potentials experienced by the constituents. This technique exploits the epithermal energy range of the neutron spectrum to achieve high wave vectors $\left(q>30 \AA^{-1}\right)$ and high energy $(\hbar \omega>1 \mathrm{eV})$ transfers. Thanks to the development of spallation neutron sources this technique was available since the beginning of 1980s, and in the last ten years, has been refined and developed, achieving appreciable performances. The DINS technique is effectively performed on inverse geometry instruments [10], where the only effective way to select neutron energies above $1 \mathrm{eV}$ is to use nuclear resonances. This on the one hand allows energy selection up to several tens of $\mathrm{eV}$, and on the other hand poses technological problems related to the neutron counting method. Indeed, a common limitation of standard ${ }^{6} \mathrm{Li}$-glass [11] neutron counters is the $1 / v$ dependence of their efficiency, $v$ being the neutron velocity. However for an inverse geometry instrument there is the possibility to use the so-called Resonance Detector (RD)

\footnotetext{
* Corresponding author. Tel.: +3906 7259 4440; fax: + 39062023507. E-mail address: alessandra.filabozzi@roma2.infn.it (A. Filabozzi).
}

configuration [12-28], where the neutron detection is realized by using a system composed by an analyzer foil and a photon detector. The RD configuration overcomes the efficiency loss of the Li-based neutron counters, providing a counting efficiency that is independent of neutron energy. This configuration has been revised and extensively tested on both eVS and VESUVIO spectrometers [29] at ISIS since 2000 , producing very good results in terms of signal to background ratio and counting efficiency [30], as already shown in many experimental works (see, e.g. Refs. [25-27]). It is important to stress that RD configuration allows, in principle, to exploit final neutron energies of several tens of $\mathrm{eV}$, such as for example the ones selectable through an uranium analyzer or other materials (e.g. ${ }^{139} \mathrm{La}$ ).

In Ref. [27], it was briefly discussed, at a conceptual level, the possibility to exploit an optimized detector configuration, in order to access a wider region of the $(\hbar \omega, \hbar q)$ kinematical space with respect to that usually spanned in DINS experiments [4], exploiting final neutron energies lying above $10 \mathrm{eV}$. Access to values $\hbar \omega \approx 50 \mathrm{eV}$ and $q \approx 100 \AA^{-1}$ at the maximum of the recoil peak would allow one to reduce the contribution of the final state effects [31-33].

A recent development for DINS measurements on the VESUVIO spectrometer, namely the Foil Cycling Technique (FCT), was found to improve the spectrometer resolution, providing an appreciable counting efficiency [34,35] and a good neutron and gamma background subtraction method.

The more recent issue to be addressed in DINS experiments is to get information on the details of the momentum distribution, 
$n(p)$, in the target constituent. This distribution reflects the microscopic local environment and thus the effective potential that the target constituent experiences. In order to study the details of $n(p)$, a good statistical quality of data is needed.

This paper aims to show the possibility to use resonance analyzers to access energy and wave vector transfers high enough for the final state effects to be reduced to a negligible level. Within this energy and wave vector regime, spectra at different scattering angles (or at the same angle but corresponding to different final neutron energies) could be properly summed up, thus allowing an enhancement of the statistics and a more reliable extraction of the momentum distribution $n(p)$ of the target constituents. In order to verify this possibility, simulations of neutron scattering off protons in water has been performed with and without the insertion of the final state effects, estimated in the framework of the additive approximation [36].

In this study, the VESUVIO spectrometer is considered as a reference $\mathrm{RD}$ time of flight spectrometer and it is used as a "laboratory" to investigate further developments.

This paper is organized as follows: in Section 2, the theoretical framework for DINS is presented, while in Section 3 the basic principles of the Resonance Detector configuration are discussed. In Section 4, Monte Carlo simulation of time of flight spectra acquired with an inverse geometry instrument from water are presented, in order to find good operational conditions for high performance DINS measurements. In Section 5, the results are discussed and the conclusions drawn.

\section{DINS theoretical framework}

The Deep Inelastic Neutron Scattering (DINS) is a unique technique providing information on the short-time dynamics of nuclei in condensed matter $[1,2,37]$. In the DINS regime, the impulse approximation (IA) holds with a good degree of accuracy, so that the dynamic structure factor is given by [2]

$S_{I A}(\mathbf{q}, \omega)=\hbar \int n(\mathbf{p}) \delta\left[\hbar \omega-\hbar \omega_{r}-\frac{\mathbf{p} \cdot \hbar \mathbf{q}}{M}\right] d \mathbf{p}$.

Eq. (1) states that scattering occurs between the neutron and a single particle of momentum $\mathbf{p}$ and mass $M$, with conservation of kinetic energy and momentum of the (particle + neutron) system. The term $\hbar \omega_{r}=\hbar^{2} q^{2} / 2 M$ is the recoil energy, i.e. the kinetic energy of a struck particle that is at rest before the collision and absorbs all the momentum transferred by the neutron.

Within the framework of the IA, $\hbar \omega$ and $q$ are explicitly coupled through the West scaling variable [38] $y=\left(M / \hbar^{2} q\right)\left(\hbar \omega-\hbar \omega_{r}\right)$, so that Eq. (1) can be reduced to the form

$S_{I A}(\mathbf{q}, \omega)=\frac{M}{\hbar q} J(y, \hat{q})$

where

$J(y, \hat{q})=\hbar \int n(\mathbf{p}) \delta(\hbar y-\mathbf{p} \cdot \hat{q}) d \mathbf{p}$.

$J(y, \hat{q})$ being the Neutron Compton Profile (NCP), formally defined as the Radon transform [39] of the momentum distribution. The quantity $\hat{q}$ is a unit vector, as $J(y, \hat{q})$ no longer depends on the magnitude of q. The function $J(y, \hat{q}) d y$ is the probability for an atom to have a momentum parallel to $\hat{q}$ of magnitude between $\hbar y$ and $\hbar(y+d y)$.

For an isotropic system the dependence on $\hat{q}$ can be dropped, and Eq. (3) becomes

$J(y)=2 \pi \hbar \int_{|\hbar y|}^{\infty} p n(p) d p$.
In actual experiments, the asymptotic formula of Eq. (1) is never attained, since IA implies the limit $(\hbar \omega, \hbar q) \rightarrow \infty$, keeping $y$ constant. For this reason Eq. (2) is generally replaced by

$S(\mathbf{q}, \omega)=\frac{M}{\hbar q} F(y, \mathbf{q})$

where the scaling function $F(y, \mathbf{q})$ retains an additional dependence on q, irreducible to the simple West scaling. Eq. (5) takes into account that the final state of the struck particle is not the free particle one (a plane wave). Such a dependence is generally known [1] as Final State Effects (FSE). Various methods for the approximate evaluation of FSE have been devised so far [31-33,40-42], the most widely used being the so-called additive approach [42].

It can be shown that within the so-called Gaussian Approximation [43], the additive approach leads to the following expression for $F(y, \mathbf{q})[36]$ :

$$
\begin{aligned}
F(y, \mathbf{q}) \simeq & {\left[1+\frac{A_{3}(\mathbf{q})}{\left(2 \sigma^{2}(\hat{\mathbf{q}})\right)^{3 / 2}} H_{3}\left(y\left(2 \sigma^{2}(\hat{\mathbf{q}})\right)^{-1 / 2}\right)\right.} \\
& +\frac{A_{4}(\mathbf{q})}{\left(2 \sigma^{2}(\hat{\mathbf{q}})\right)^{2}} H_{4}\left(y\left(2 \sigma^{2}(\hat{\mathbf{q}})\right)^{-1 / 2}\right) \\
& \left.+\frac{\left(A_{3}(\mathbf{q})\right)^{2}}{2\left(2 \sigma^{2}(\hat{\mathbf{q}})\right)^{3}} H_{6}\left(y\left(2 \sigma^{2}(\hat{\mathbf{q}})\right)^{-1 / 2}\right)\right] J(y, \hat{\mathbf{q}})
\end{aligned}
$$

where $H_{m}\left(y\left(2 \sigma^{2}(\hat{\mathbf{q}})\right)^{-1 / 2}\right)$ are the $m$ th order Hermite polynomials and $\sigma^{2}(\hat{\mathbf{q}})$ is the second moment of $J(y, \hat{\mathbf{q}})$. As for Eq. (3), the dependence on $\widehat{\mathbf{q}}$ in Eq. (6) can be dropped for an isotropic system. The terms $A_{3}$ and $A_{4}$ in Eq. (6) are given by [36]

$$
\begin{aligned}
& A_{3}(\mathbf{q})=\frac{M}{12 \hbar^{2} q}\left\langle\sum_{j, k=1}^{3} \hat{q}_{j} \frac{\partial^{2} V}{\partial r_{j} \partial r_{k}} \hat{q}_{k}\right\rangle \\
& A_{4}(\mathbf{q})=\frac{M^{2}}{24 \hbar^{4} q^{2}}\left\langle\left(\sum_{j=1}^{3} \hat{q}_{j} \frac{\partial V}{\partial r_{j}}\right)^{2}\right\rangle
\end{aligned}
$$

$V$ being the potential energy of the system, $\vec{r}$ being the struck particle coordinate and $\langle\ldots\rangle$ the quantum-statistical average, including the sum over all the nuclei. In this work we use the additive approach [36] to estimate FSE. From Eq. (6) it is clear that at high momentum transfer the scaling function $F(y, \mathbf{q})$ does not depend any more on the magnitude of $\mathbf{q}$ and reduces to $J(y, \hat{q})$.

\section{The resonance detector configuration}

The RD counting procedure relies upon two main steps $[23,24]$. In the first one, the scattered neutron beam impinges onto the analyzer foil which provides the energy analysis by means of $(\mathrm{n}, \gamma)$ resonance absorption at a given resonance energy $E_{r}$. In the second step, the prompt gamma-rays are detected to assign the total neutron time of flight.

It has to be stressed that the gamma detector is used as a counter: for each absorbed neutron into the analyzer, the production of a gamma-ray cascade occurs. It is enough that one photon of the cascade is detected, even by a partial release of its energy in the detector's active medium, to trigger a counting signal.

The experimental signal recorded in the RD configuration is a time of flight spectrum, representing the number of counts collected in a time channel of width $\delta t$ centered in $t$. The count rate per time bin is given by the expression [36]

$C(t,\langle 2 \vartheta\rangle)=\beta \cdot I_{P} \int_{0}^{\infty} d E_{0} \Phi\left(E_{0}\right) \int_{0}^{\infty} d E_{1} T\left(E_{1}\right) \delta(\tau)\left(\frac{d^{2} \sigma}{d \Omega d \hbar \omega}\right)$ 
where

$\beta=n_{T} \Delta \Omega \rho_{s} d_{s} \eta$

$I_{P}=\int_{-\infty}^{\infty} d t_{0} \int_{0}^{\pi} d\left(2 \vartheta^{\prime}\right) \int_{0}^{\infty} d L_{0}^{\prime} \int_{0}^{\infty} d L_{1}^{\prime} P\left(t_{0}, 2 \vartheta^{\prime}, L_{0}^{\prime}, L_{1}^{\prime}\right)$

the argument in the $\delta$-function being

$\tau=t-t_{0}-L_{0} \sqrt{\frac{m_{n}}{2 E_{0}}}-L_{1} \sqrt{\frac{m_{n}}{2 E_{1}}}$

with $m_{n}$ the neutron mass. In the previous Eq. (8), the quantities $\Phi\left(E_{0}\right), T\left(E_{1}\right)$ and $d^{2} \sigma / d \Omega d \hbar \omega$ are the neutron flux at the incident neutron energy, the analyzer's energy transfer function and the double differential scattering cross-section of the sample, respectively, while $E_{0}$ and $E_{1}$ are the initial ad final neutron energies, respectively. The quantity $n_{T}$ in Eq. (9) is the number of neutron pulses included in the measurement, $\Delta \Omega$ the solid angle defining the detector acceptance, $\eta$ the overall detection efficiency, while $\rho_{s}$ and $d_{s}$ are the sample's density and thickness, respectively. In Eq. (10), $P\left(t_{0}, 2 \vartheta, L_{0}, L_{1}\right)$ is the probability distribution that a given neutron leaves the moderator with a time-delay $t_{0}$, travels from the moderator to the sample along a flight path $L_{0}$, is scattered at an angle $2 \vartheta$, and eventually travels from the sample to the detector along a flight path $L_{1}$.

The wide photon spectrum from the analyzer (atomic X-rays and nuclear gamma-rays) allows one to use energy discrimination thresholds that improve the $S / B$ ratio, still guaranteeing an acceptable counting efficiency [26].

Eq. (8) represents only the signal component, i.e. it assumes the absence of background. A more complete expression should contain background terms, as already discussed in previous experimental papers $[44,45]$. The count rate of Eq. (8) can be expressed, for each recoiling mass $M_{i}(i=1,2, \ldots N)$ presents in the scattering sample, in terms of the corresponding scaling function $F_{M_{i}}(y, \mathbf{q})$. Since

$$
\frac{d^{2} \sigma}{d \Omega d \hbar \omega}=\sum_{i=1}^{N} \sqrt{\frac{E_{1}}{E_{0}}} \frac{M_{i} b_{i}^{2}}{\hbar^{2} q} F_{M_{i}}\left(y_{i}, \mathbf{q}\right)
$$

where $N$ is the number of different recoiling particles present in the scatterer and $y_{i}$ the West scaling variable of the $i$ th recoiling particle, and $b_{i}$ its scattering length, then the total count rate becomes

$C(t,\langle 2 \vartheta\rangle)=\beta \sum_{i=1}^{N} I_{P} \int_{0}^{\infty} d E_{0} \Phi\left(E_{0}\right) \int_{0}^{\infty} d E_{1} T\left(E_{1}\right) \delta(\tau) \sqrt{\frac{E_{1}}{E_{0}}} \frac{M_{i} b_{i}^{2}}{\hbar^{2} q} F_{M_{i}}\left(y_{i}, \mathbf{q}\right)$

Given Eq. (13), in the time of flight spectrum there will appear $N$ recoil peaks (one per each recoiling mass), whose intensities are proportional to the number of atoms contained in the sample, weighted by the scattering cross-section. The time width and the shape of each recoil peak depend on the width and shape of $F_{M i}\left(y_{i}, \mathbf{q}\right)$, on the uncertainties of the geometrical parameters $L_{0}, L_{1}$, 29 and of the time of flight $t$, and on the width and shape of the analyzer's transfer function $T\left(E_{1}\right)$. The width of $T\left(E_{1}\right)$ can be reduced, still maintaining an acceptable count rate, by applying the FCT, as already discussed in previous experimental papers [34,35].

\section{Monte Carlo simulations}

The Monte Carlo code employed to simulate the DINS time of flight spectra is a modified version of the DINSMS code, developed by Mayers et al. [46] for DINS measurements on VESUVIO spectrometer when operating in the so-called Resonance Filter (RF) or Filter Difference (FD) configuration [47]. The code takes into account the specific features of the neutron pulsed source, the filters and the detectors present on VESUVIO. In the Monte Carlo simulation, the result is a sum of different neutron histories, weighted according to the probabilities that would occur in a real experiment. In Ref. [46], a more detailed description of the code is provided.

The neutron transport code has been modified in order to incorporate the code modules for neutron detection in the RD configuration. The modules describing the spectrometer components have been chosen to be, at a first stage, identical to a real experimental condition [27]. Then a study of the time of flight spectrum in different geometrical configurations (detectors positions, primary and scattering flight paths, $L_{0}$ and $L_{1}$ ), in combination with different kind of analyzer foils has been undertaken. Our aim is to investigate the possibility that in recoil peaks at different scattering angles, the contribution of the final state effects are negligible, so that the different peaks can be considered together and the corresponding scaling function $F(y, \mathbf{q})$ summed up thus obtaining a better statistics.

\subsection{Simulations in the standard $R D$ configuration in $I A$}

The first set of simulations have been carried out considering the spectrometer in the standard RD configuration, with an array of YAP scintillators [27] as neutron counters. In the simulations the scattering angle $2 \vartheta$ is considered in the range $15^{\circ} \leq 2 \vartheta \leq 60^{\circ}$, somewhat wider than the usual range in VESUVIO [4]. The instrumental parameters were $L_{0}=11.055 \mathrm{~m},\left\langle L_{1}\right\rangle=0.746 \mathrm{~m}$, $\left\langle L_{1}\right\rangle$ being the scattering flight path averaged over the whole set of scattering angle in the array (i.e. $20^{\circ} \leq 2 \vartheta \leq 60^{\circ}$ ). The sample was a slab of $5.0 \mathrm{~cm}$ height, $5.0 \mathrm{~cm}$ width and $0.1 \mathrm{~cm}$ thickness, chosen in order to fit the beam dimension whose actual diameter is about $4.5 \mathrm{~cm}$ (umbra+penumbra). The analyzers were chosen to be a ${ }^{238} \mathrm{U}$ or ${ }^{139} \mathrm{La}$ metallic foils. These nuclides possess radiative neutron capture resonances at about $6.67,20.8$, 36.6 and $66.0 \mathrm{eV}$ (uranium) and $72.0 \mathrm{eV}$ (lanthanum). Fig. 1 shows

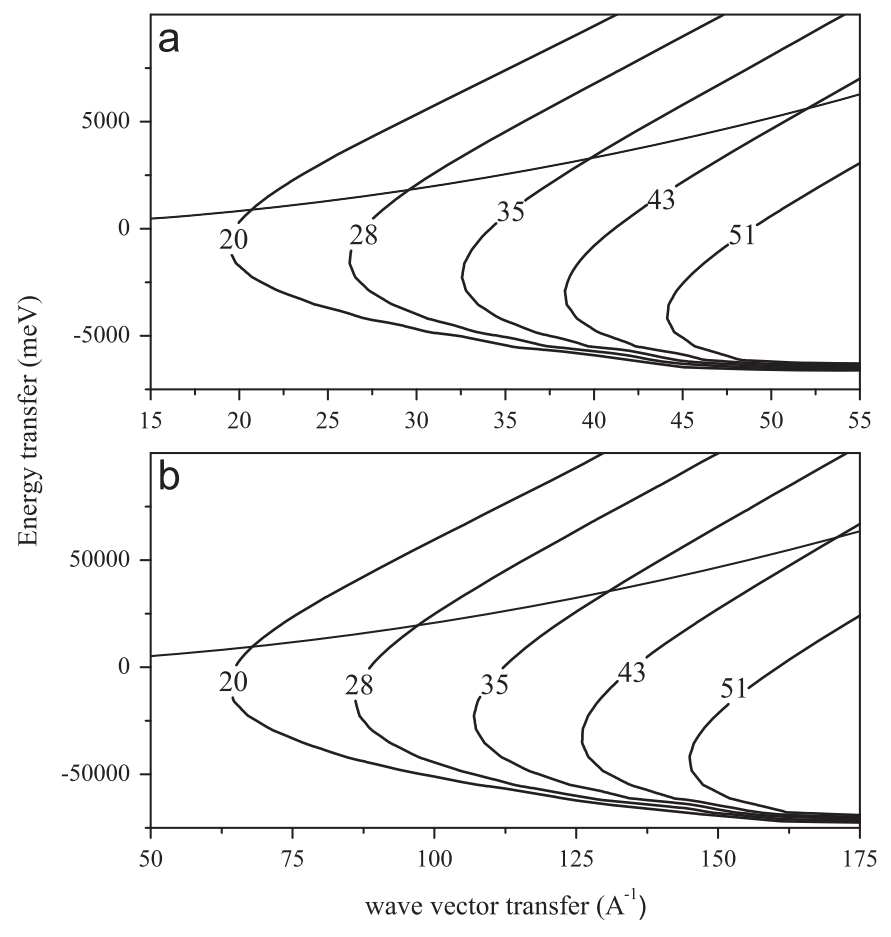

Fig. 1. Region of kinematical space (wave vector transfers and energy transfers) accessible by employing a $6.67 \mathrm{eV}$ (a) and a $72.0 \mathrm{eV}$ (b) resonance for five typical values of the scattering angles $\left(2 \vartheta^{\circ}=20^{\circ}, 28^{\circ}, 35^{\circ}, 43^{\circ}\right.$ and $\left.51^{\circ}\right)$ for forward scattering experiments. The continuous line intercepting the iso-angular lines is the proton recoil line $\hbar \omega=\hbar \omega_{r}$. 
the regions of the kinematical space accessible by selecting a $6.67 \mathrm{eV}$ resonance (panel (a)) or a $72.0 \mathrm{eV}$ resonance (panel (b)), both for typical values of the scattering angles for forward scattering experiments. The negative region of $\hbar \omega$ correspond to events where the neutron gain energy in the scattering event from the struck atom (energy gain). The continuous line, intercepting the iso-angular lines, is the proton recoil line $\hbar \omega_{r}=$ $\hbar^{2} q^{2} / 2 M$. From Fig. 1 it is clear that for the same scattering angle much higher energy and wave vector transfers can be achieved with an higher resonance energy.

In the standard RD configuration, the transfer function of the analyzer foil is given by

$T(E)=1-\exp (-d / \lambda(E))$

$d$ being the foil thickness, $\lambda(E)$ the mean free path given by

$\lambda(E)=\frac{A}{N_{A} \rho \sigma_{(n, \gamma)}(E)}$

where $A$ is the mass number of the absorbing nucleus, $N_{A}$ the Avogadro's number, $\rho$ the density and $\sigma_{(n, \gamma)}(E)$ the analyzer's radiative capture cross-section. Fig. 2 shows the energy transfer function, $T_{R D}(E)$, for an uranium foil $30 \mu \mathrm{m}$ thickness at the four different values of the resonance energy. This thickness, corresponding to fractions between 0.3 and 0.9 of the neutron interaction length for the different resonance energies, guarantees that the thin foil approximation (TFA) [17,35] holds with high degree of accuracy for the whole set of resonances. The TFA provides a good compromise between counting efficiency and resolution, as already shown in previous experimental papers [17,35]. In Table 1, the main parameters characterizing each $T(E)$ are listed. From Eq. (13), it is clear that the intensity and the line shape of the DINS recoil spectrum in the scattering signal $\langle C(t, 2 \vartheta)\rangle$, apart the contribution of the FSE, depend on the functional form of $J(y)$. The latter is given as an input for the Monte Carlo code and it was chosen to be of Gaussian form, namely

$J(y)=\frac{1}{\sqrt{2 \pi \sigma^{2}}} \cdot \exp \left(-y^{2} / 2 \sigma^{2}\right)$

A $\mathrm{H}_{2} \mathrm{O}$ molecular system was chosen as a test sample. The value of $\sigma$ was fixed at $4.841 \AA^{-1}$, as experimentally determined in previous DINS measurements at VESUVIO [48], corresponding to a proton mean kinetic energy $\left\langle E_{k}\right\rangle=145 \mathrm{meV}$.

Fig. 3 shows simulated DINS time of flight spectra for detectors placed at scattering angles of $60.7^{\circ}$ and $48.7^{\circ}$ (a) and at $35^{\circ}$ and $20^{\circ}$ (b), considering four final neutron energies: $6.67 \mathrm{eV}, 20.8 \mathrm{eV}$, $36.6 \mathrm{eV}$ and $66.0 \mathrm{eV}\left({ }^{238} \mathrm{U}\right.$ resonances). The larger peaks located at different tof positions in the two panels are the hydrogen recoil signals. The less intense ${ }^{16} \mathrm{O}$ recoil signals are found at $325 \mu \mathrm{s}$, $180 \mu \mathrm{s}, 140 \mu \mathrm{s}$ and $105 \mu \mathrm{s}$ for the final neutron energies quoted above in both the panels, respectively.

It has to be noted that at high scattering angles (a), due the combined effects of geometry and resolution, the ${ }^{16} \mathrm{O}$ recoil peaks cannot be clearly identified and subtracted, since they either lye beneath the more intense ${ }^{1} \mathrm{H}$ recoil peaks or are not well separated.

From the panel (b) in Fig. 3, it can be noted that only for the $6.67 \mathrm{eV}$ resonance (i.e. $t \simeq 325 \mu$ s) the ${ }^{1} \mathrm{H}$ and ${ }^{16} \mathrm{O}$ peaks are completely overlapped at very low angles.

The simulation shows that for scattering angles between $25^{\circ}$ and $35^{\circ}$, all peaks in the time of flight spectra are sufficiently well separated for the whole set of final neutron energies considered.

In order to obtain a clearer separation of the recoil peaks, different geometrical configuration are explored. First, the effect of $L_{0}$ on the whole instrument response is studied, changing $L_{0}$ from $11 \mathrm{~m}$ to $25 \mathrm{~m}$, while fixing $\left\langle L_{1}\right\rangle$ at the same value $(0.746 \mathrm{~m})$ of the previous simulations. Indeed, it is well known that one of the contributions to the overall spectrometer resolution function

Table 1

Main analyzer parameters in the RD configuration. $E_{r}$ is the resonance energy FWHM the Full Width at Half Maximum of the transfer function $T(E), I_{0}$ the peak value of $T(E)$ and $d_{A} / \lambda\left(E_{r}\right)$ the ratio of the analyzer's thickness to the mean interaction length calculated at a given resonance energy.

\begin{tabular}{rlll}
\hline$E_{r}(\mathrm{meV})$ & FWHM (meV) & $I_{0}$ & $d_{A} / \lambda\left(E_{r}\right)$ \\
\hline 6671 & 152 & 0.53 & 0.80 \\
20,800 & 230 & 0.58 & 0.87 \\
36,600 & 347 & 0.52 & 0.78 \\
66,000 & 340 & 0.26 & 0.30 \\
72,000 & 540 & 0.65 & 1.0 \\
\hline
\end{tabular}
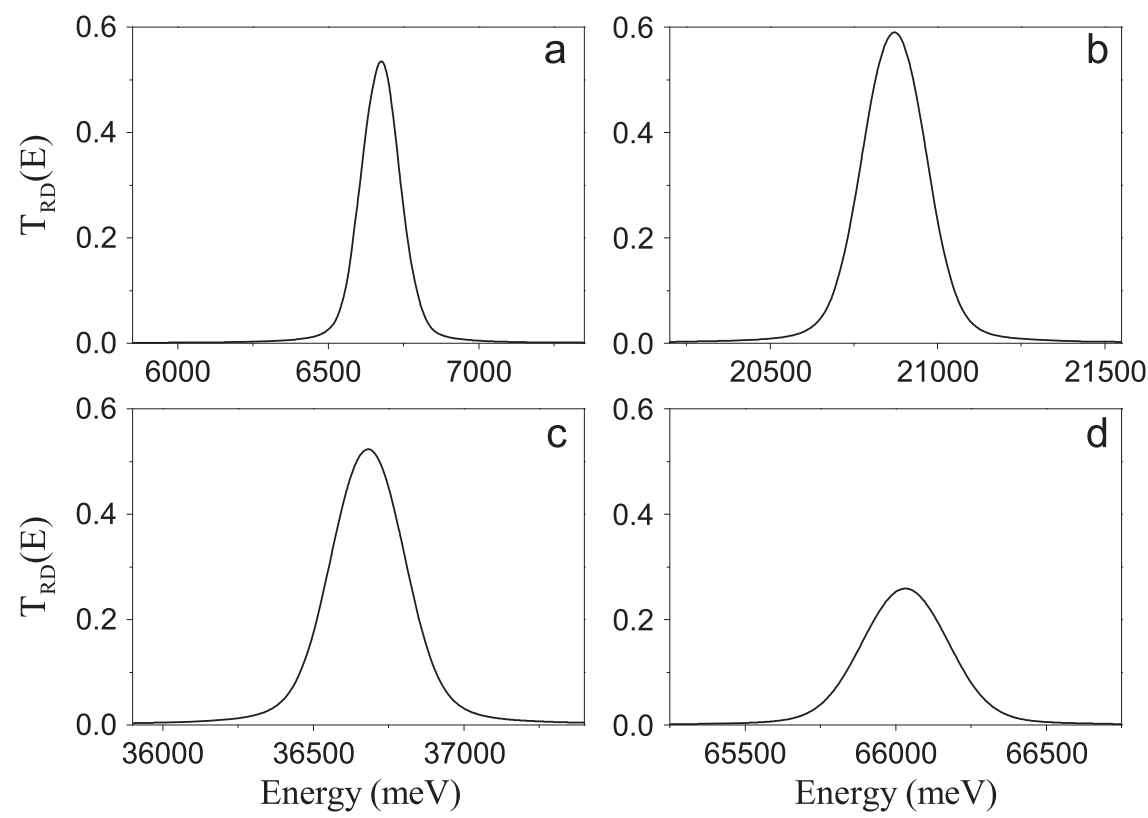

Fig. 2. Energy transfer function, $T_{R D}(E)$, in the RD configuration for an uranium foil $30 \mu \mathrm{m}$ thickness at four different values of the resonance energy, namely $6.67 \mathrm{eV}$ (a), $20.8 \mathrm{eV}(\mathrm{b}), 36.6 \mathrm{eV}$ (c) and $66.0 \mathrm{eV}$ (d), respectively. 


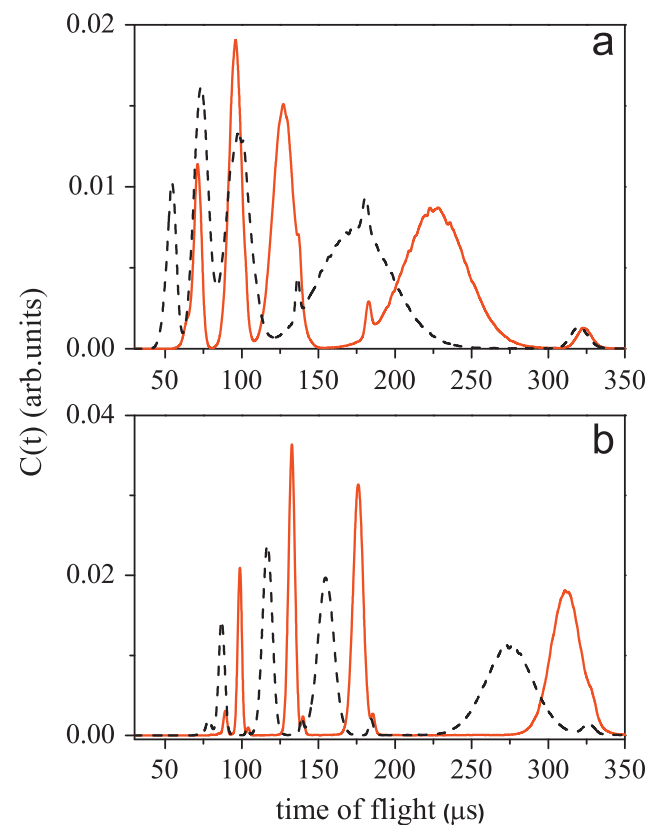

Fig. 3. Simulated DINS time of flight spectra for an $\mathrm{H}_{2} \mathrm{O}$ molecular system, for the four ${ }^{238} \mathrm{U}$ resonances at $6.67 \mathrm{eV}, 20.8 \mathrm{eV}, 36.6 \mathrm{eV}$ and $66.0 \mathrm{eV}$ and a flight path $L_{0}=11.0 \mathrm{~m}$ with detectors placed at: (a) scattering angles of $60.7^{\circ}$ (dashed line) and $48.7^{\circ}$ (solid line); (b) scattering angles of $35^{\circ}$ (dashed line) and $20^{\circ}$ (solid line).

depends on the $L_{1} / L_{0}$ ratio $[49,50]$ and that an higher $L_{0}$ gives a better resolution. Fig. 4 shows the time of flight spectra obtained for a $\mathrm{H}_{2} \mathrm{O}$ sample, employing the same $J(y)$ given in Eq. (16), for detectors at scattering angles between $15^{\circ}$ and $40^{\circ}$. From Fig. 4(a), it can be noted that for detectors at $15^{\circ}$ the ${ }^{16} \mathrm{O}$ and ${ }^{1} \mathrm{H}$ recoil peaks relative to the $6.67 \mathrm{eV}$ and $20.8 \mathrm{eV}{ }^{238} \mathrm{U}$ resonances are mixed, while at $40^{\circ}$, the ${ }^{16} \mathrm{O}$ and ${ }^{1} \mathrm{H}$ recoil peaks corresponding to 20.8 and $36.6 \mathrm{eV}$ final neutron energies overlap. On the other hand, the separation of the ${ }^{1} \mathrm{H}$ and ${ }^{16} \mathrm{O}$ peaks at $20^{\circ}$ and $30^{\circ}$, shown in Fig. 4(b), is satisfactory for almost all final neutron energies.

The effect of a different final neutron energy selection has been also investigated by using the $72 \mathrm{eV}$ resonance energy of a ${ }^{139} \mathrm{La}$ analyzer foil. Fig. 5 shows the time of flight spectra for $\mathrm{H}_{2} \mathrm{O}$ relative to a gaussian $J(y)$ for $L_{0}=11.0$ (a) and $25.0 \mathrm{~m}$ (b), respectively, and for different scattering angles $\left(20^{\circ}\right.$ and $\left.40^{\circ}\right)$. In the two panels, the small peaks at large tof are from ${ }^{16} \mathrm{O}$, while the large ones at low tof are from ${ }^{1} \mathrm{H}$. In both cases the ${ }^{1} \mathrm{H}$ and ${ }^{16} \mathrm{O}$ peaks are well separated also at high scattering angles. For the longer primary flight path case, the DINS time of flight spectra put in evidence a clear peak separation down to about $20^{\circ}$.

\subsection{Simulations in the RD configuration with FSE}

The same set of simulations on $\mathrm{H}_{2} \mathrm{O}$ described before are performed taking into account the FSE, in the framework of the Gaussian Approximation+additive approach, as described in Section 2, and employing, in a very simplified model, a purely harmonic potential.

In what follows we present and discuss simulated results obtained. Fig. 6 shows simulated DINS time of flight spectra from $\mathrm{H}_{2} \mathrm{O}$ with a ${ }^{238} \mathrm{U}$ analyzer foil and for a detector at $29=30^{\circ}$, with and without the FSE contributions, for the instrument set up with $L_{0}=11 \mathrm{~m}$ and $25 \mathrm{~m}$, in panels (a) and (b), respectively. It can be noticed that the FSE are observable in the recoil peaks only at the lowest final resonance energy $\left(t \simeq 290 \mu \mathrm{s}\right.$ for $L_{0}=11 \mathrm{~m}, t \simeq 630 \mu \mathrm{s}$

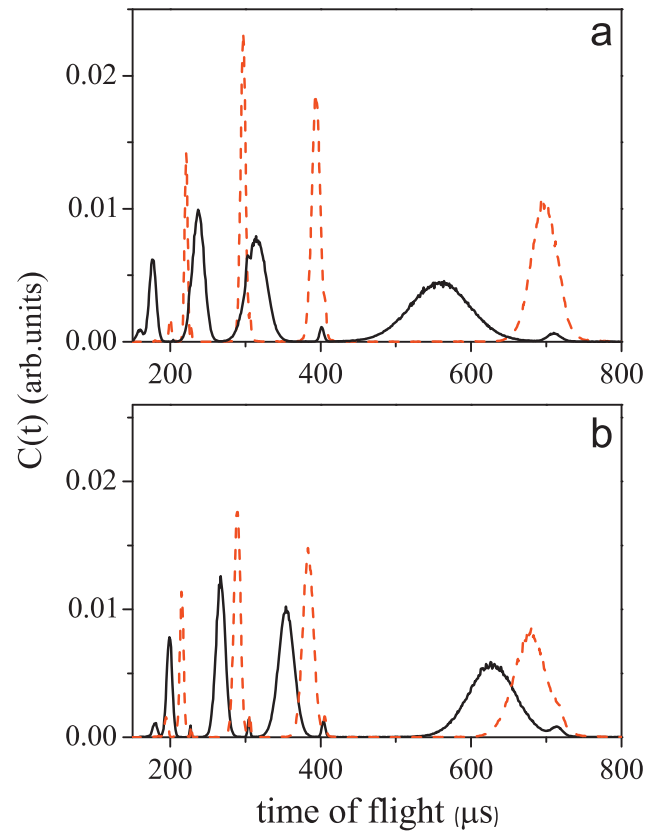

Fig. 4. Simulated DINS time of flight spectra for an $\mathrm{H}_{2} \mathrm{O}$ molecular system, considering the four ${ }^{238} \mathrm{U}$ resonances at $6.67 \mathrm{eV}, 20.8 \mathrm{eV}, 36.6 \mathrm{eV}$ and $66.0 \mathrm{eV}$ and a flight path $L_{0}=25.0 \mathrm{~m}$ with detectors placed at: (a) scattering angles of $40^{\circ}$ (solid line) and $15^{\circ}$ (dashed line); (b) scattering angles of $30^{\circ}$ (solid line) and $20^{\circ}$ (dashed line).

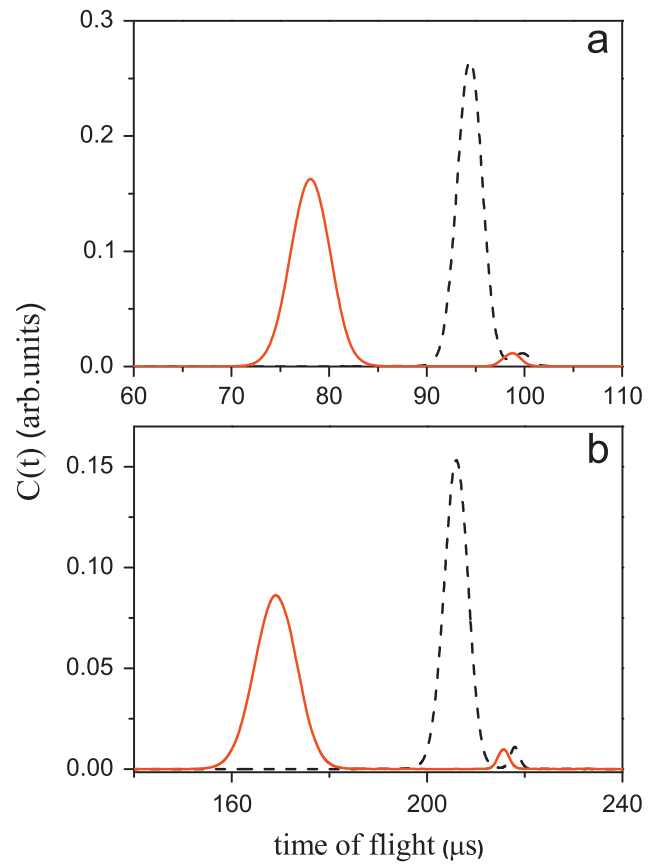

Fig. 5. Time of flight spectra for $\mathrm{H}_{2} \mathrm{O}$ relative to a gaussian $J(y)$ for a ${ }^{139} \mathrm{La}$ analyzer and for different scattering angles $20^{\circ}$ (dashed line) and $40^{\circ}$ (solid line) for (a) $L_{0}=11.0 \mathrm{~m}$ and (b) $L_{0}=25.0 \mathrm{~m}$. The ${ }^{16} \mathrm{O}$ recoil peaks are located at about $100 \mu \mathrm{s}$ for (a) and $218 \mu \mathrm{s}$ for (b), while the ${ }^{1} \mathrm{H}$ recoil profiles are located at about $94 \mu \mathrm{s}$ and $78 \mu$ s for (a) and at about $206 \mu$ s and $170 \mu$ s for (b).

for $L_{0}=25 \mathrm{~m}$ ), while they are almost negligible at higher resonance energies due to the higher $\omega$ and $q$ accessed.

Fig. 7 reports the same comparison shown in Fig. 6, but for a ${ }^{139}$ La analyzer foil.

The couples $(\hbar \omega, q)$ at the maximum of the ${ }^{1} \mathrm{H}$ recoil peaks accessed at the scattering angle shown in the figures are ordering the peaks from high-to-low tof $\left(2 \mathrm{eV}, 32 \AA^{-1}\right),\left(7 \mathrm{eV}, 57 \AA^{-1}\right)$, 


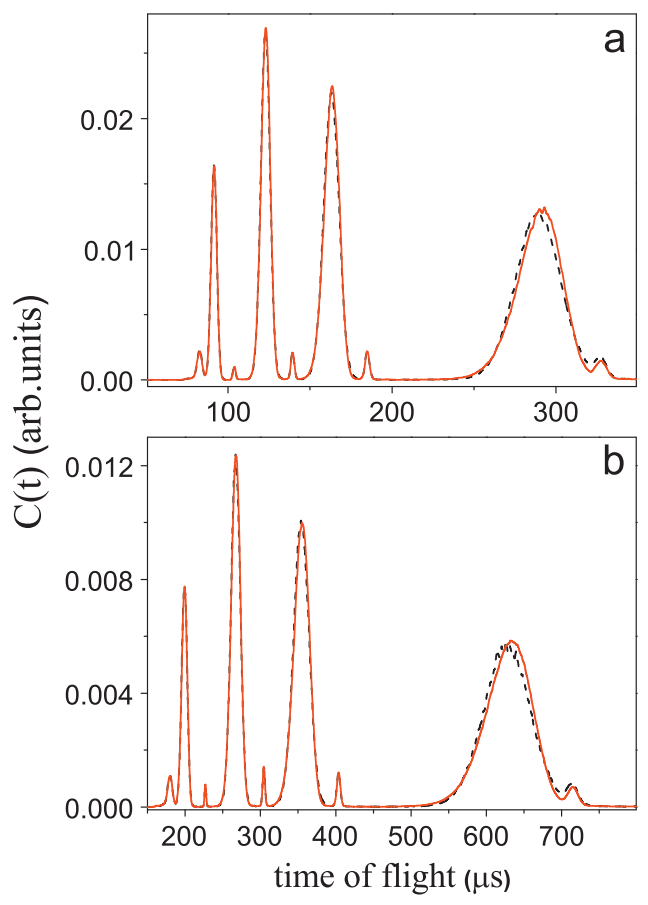

Fig. 6. Simulated DINS time of flight spectra from $\mathrm{H}_{2} \mathrm{O}$ with a ${ }^{238} \mathrm{U}$ analyzer foil for a detector at $2 \vartheta=30^{\circ}$, with FSE contributions (solid line) and without FSE contributions (dashed line) with (a) flight path $L_{0}=11.0 \mathrm{~m}$, (b) flight path $L_{0}=25.0 \mathrm{~m}$.

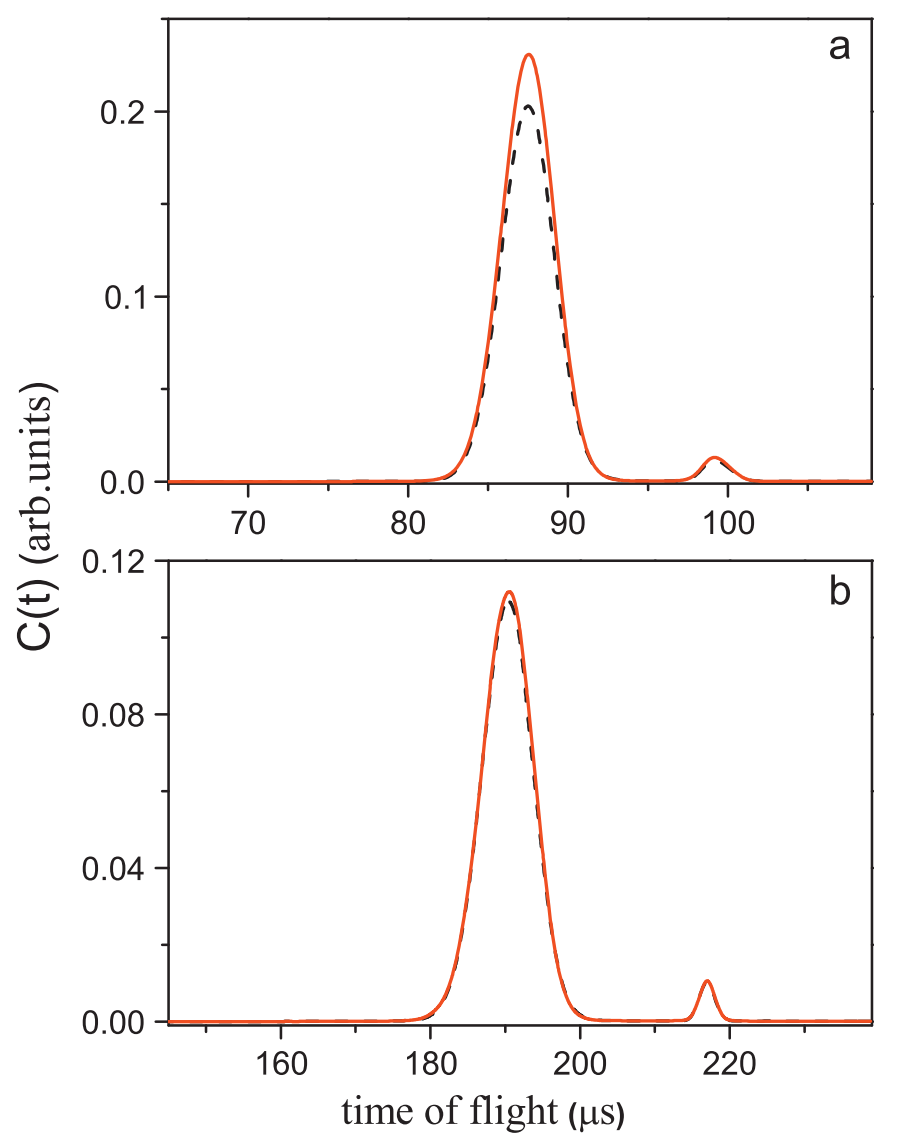

Fig. 7. Simulated DINS time of flight spectra from $\mathrm{H}_{2} \mathrm{O}$ with a ${ }^{139} \mathrm{La}$ analyzer foil and for a detector at $29=30^{\circ}$, with FSE contributions (solid line) and without FSE contributions (dashed line); with (a) flight path $L_{0}=11.0 \mathrm{~m}$, (b) flight path $L_{0}=25.0 \mathrm{~m}$. $\left(12 \mathrm{eV}, 77 \AA^{-1}\right)$ and $\left(22 \mathrm{eV}, 103 \AA^{-1}\right)$ for the case of the uranium analyzer and $\left(24 \mathrm{eV}, 108 \AA^{-1}\right)$ for the lanthanum. In the latter case, the FSE are negligible at all scattering angles, since the line shape is unaffected by FSE.

In what follows we focus our attention on the case $L_{0}=25 \mathrm{~m}$ that seems the most appropriate configuration in terms of peak separation.

\subsection{Simulations employing the foil cycling technique in IA}

A second set of simulations was carried out by changing the transfer function of the analyzer foil in order to introduce the effect of the FCT [34]. The latter relies on the use of two foils of the same absorbing material, a stationary analyzer foil and a socalled cycling foil that alternates in and out of the scattered neutron beam, thus combining the RD and the Filter Difference (FD) techniques. The double energy analysis performed in the FCT provides a substantial narrowing of the spectrometer resolution function and an effective neutron and gamma background subtraction, with only an acceptable loss in the counting efficiency [34,35].

The overall transfer function in the FCT configuration is given by [35]

$T_{F C T}(E)=\left(1-e^{-N \sigma_{(n, \gamma)}(E) d_{A}}\right) \cdot\left(1-e^{-N \sigma_{t o t}(E) d_{F}}\right)$

$d_{A}$ and $d_{F}$ being the analyzer and cycling foil thickness, respectively, $\sigma_{(n, \gamma)}$ the radiative capture cross-section and $\sigma_{t o t}(E)$ the total cross-section of the filter.

Fig. 8 shows the FCT transfer functions (dashed lines) obtained for the first four ${ }^{238} \mathrm{U}$ resonances considering $d_{A}=30 \mu \mathrm{m}$ and $d_{F}=90 \mu \mathrm{m}$. Fig. 9 shows the FCT transfer function (again dashed line) for the ${ }^{139} \mathrm{La}$ case, with $d_{A}=d_{F}=350 \mu \mathrm{m}$. In Figs. 8 and 9 the FCT transfer functions are compared with the standard RD transfer functions (solid lines).

The main characteristics of the FCT transfer functions for the two types of analyzer foil materials are listed in Table 2 for a more direct and clear comparison with the standard RD transfer functions (see Table 1).

As it can be noticed, there is an appreciable narrowing of the FWHM of the FCT transfer functions, as compared to the corresponding RD ones, between 14 and $26 \%$. The counting efficiency is still appreciable for almost all of the final neutron energies selected (even for the case of the fourth ${ }^{238} \mathrm{U}$ resonance, and of the ${ }^{139} \mathrm{La}$ resonance which are not optimized with respect to the analyzer thickness). Simulated DINS time of flight spectra for $\mathrm{H}_{2} \mathrm{O}$ have been obtained following the procedure already described in Section 4.1 for the standard RD configuration, but inserting the proper FCT transfer function in the code.

Fig. 10 shows simulated tof spectra of $\mathrm{H}_{2} \mathrm{O}$ at $20^{\circ}$ and $40^{\circ}$ with $L_{0}=25 \mathrm{~m}$ for ${ }^{238} \mathrm{U}$ and ${ }^{139} \mathrm{La}$ analyzers in panels (a) and (b), respectively. The ${ }^{1} \mathrm{H}$ and ${ }^{16} \mathrm{O}$ recoil peaks are in the same positions of those shown in Fig. 4 and for a given $(\hbar \omega, q)$ couple, but the peaks in the present case are narrower due to the effect of $T_{F C T}(E)$. Although the better resolution, the peak separation is not sufficiently good for all the resonances. In fact, it can be observed that in the case of uranium (panel (a)), the peak separation is good and for all the resonances with the exception of the first ${ }^{1} \mathrm{H}$ peak for $29=20^{\circ}$ (at about $700 \mu \mathrm{s}$ ) and of the second and the third one (at about $320 \mu$ s and $250 \mu$ s, respectively) for $29=40^{\circ}$. In the case of lanthanum (panel (b)), the peak separation is quite good at all the scattering angles.

\section{4. y domain analysis}

Analysis of the DINS data is made typically in the $y$ domain rather than in the TOF one. Due to the scaling properties, the 


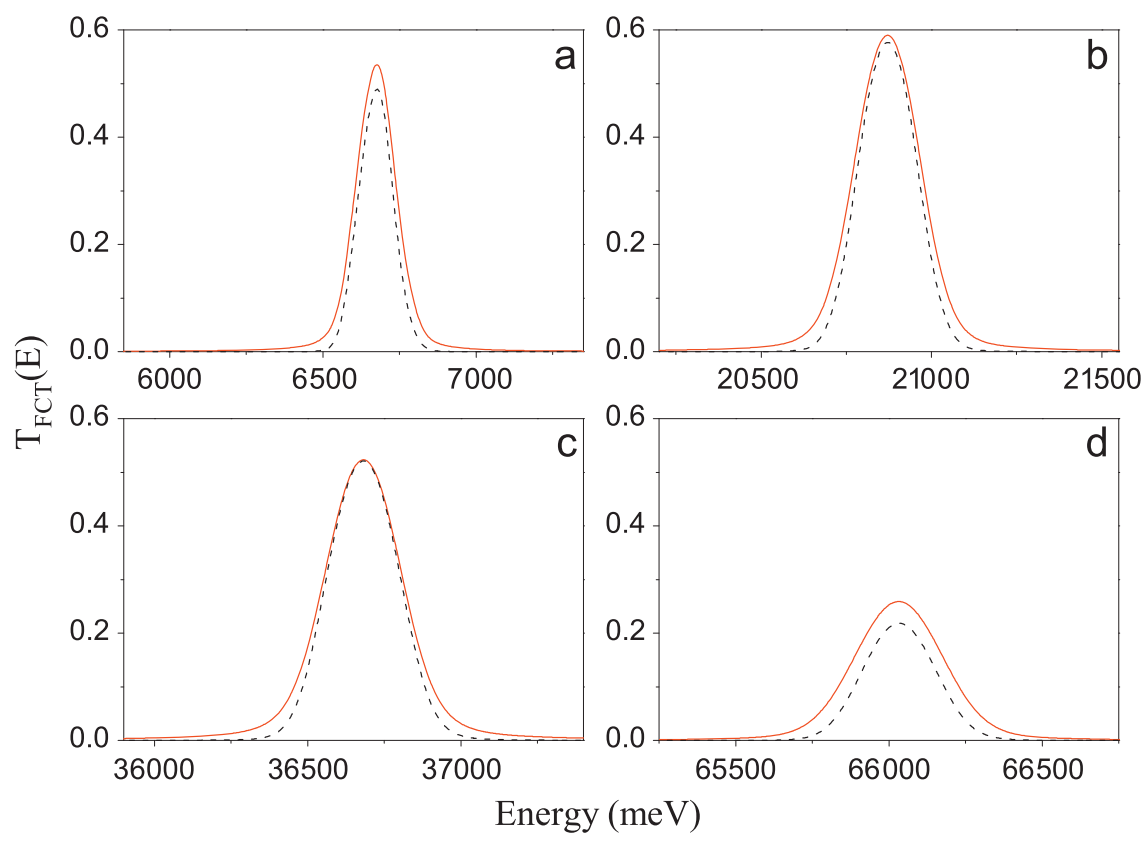

Fig. 8. FCT transfer functions obtained for the first four ${ }^{238} \mathrm{U}$ resonances at $6.67,20.8,36.6$ and $66.0 \mathrm{eV}$ (panels a, b, c, $\mathrm{d}$ respectively), considering $d_{A}=30 \mu \mathrm{m}$ and $d_{F}=90 \mu \mathrm{m}$. Solid lines represent the standard RD transfer functions, dashed lines the FCT transfer functions.

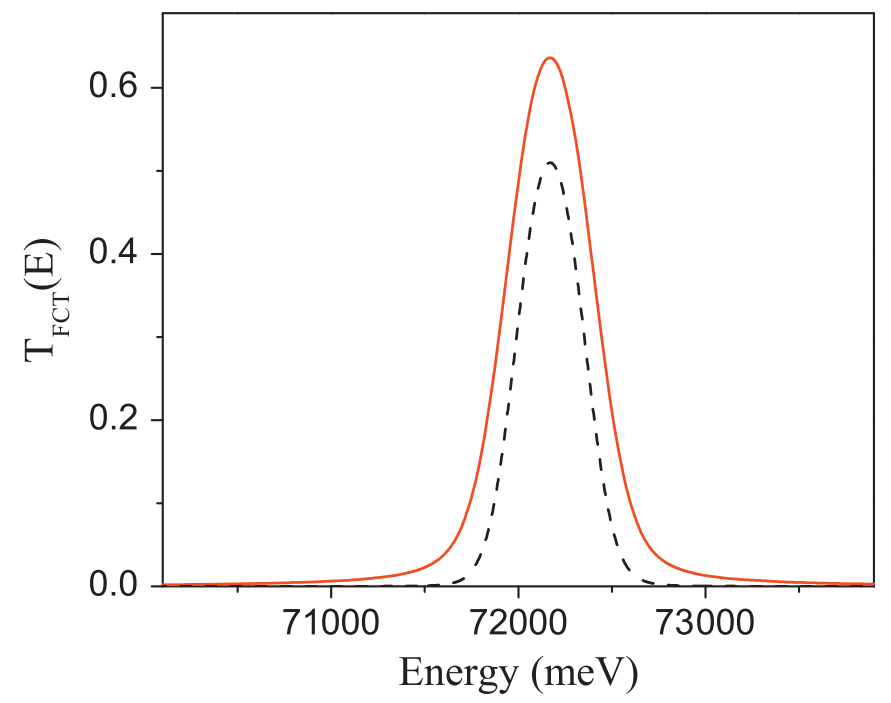

Fig. 9. FCT transfer function for the ${ }^{139}$ La case, with $d_{A}=d_{F}=350 \mu \mathrm{m}$. Solid line represents the standard RD transfer function, dashed line represents the FCT transfer function.

Table 2

Main analyzer parameters in the FCT configuration. $E_{r}$ is the resonance energy: FWHM the Full Width at Half Maximum of the transfer function $T(E) ; I_{0}$ the peak value of $T(E)$; and $\beta=I_{0}^{F C T} / I_{0}^{R D}$.

\begin{tabular}{rlll}
\hline$E_{r}(\mathrm{meV})$ & FWHM $(\mathrm{meV})$ & $I_{0}$ & $\beta$ \\
\hline 6671 & 126 & 0.49 & 0.92 \\
20,800 & 198 & 0.57 & 0.98 \\
36,600 & 265 & 0.52 & 1.0 \\
66,000 & 262 & 0.22 & 0.85 \\
72,000 & 400 & 0.51 & 0.78 \\
\hline
\end{tabular}

scaling function $F(y)$ is centered in $y=0$ and is symmetric. The effect of the final state interactions produces a shift of the recoil peak maximum towards negative values of $y$ as well as asymmetries and distortions due to the presence of terms that are odd in

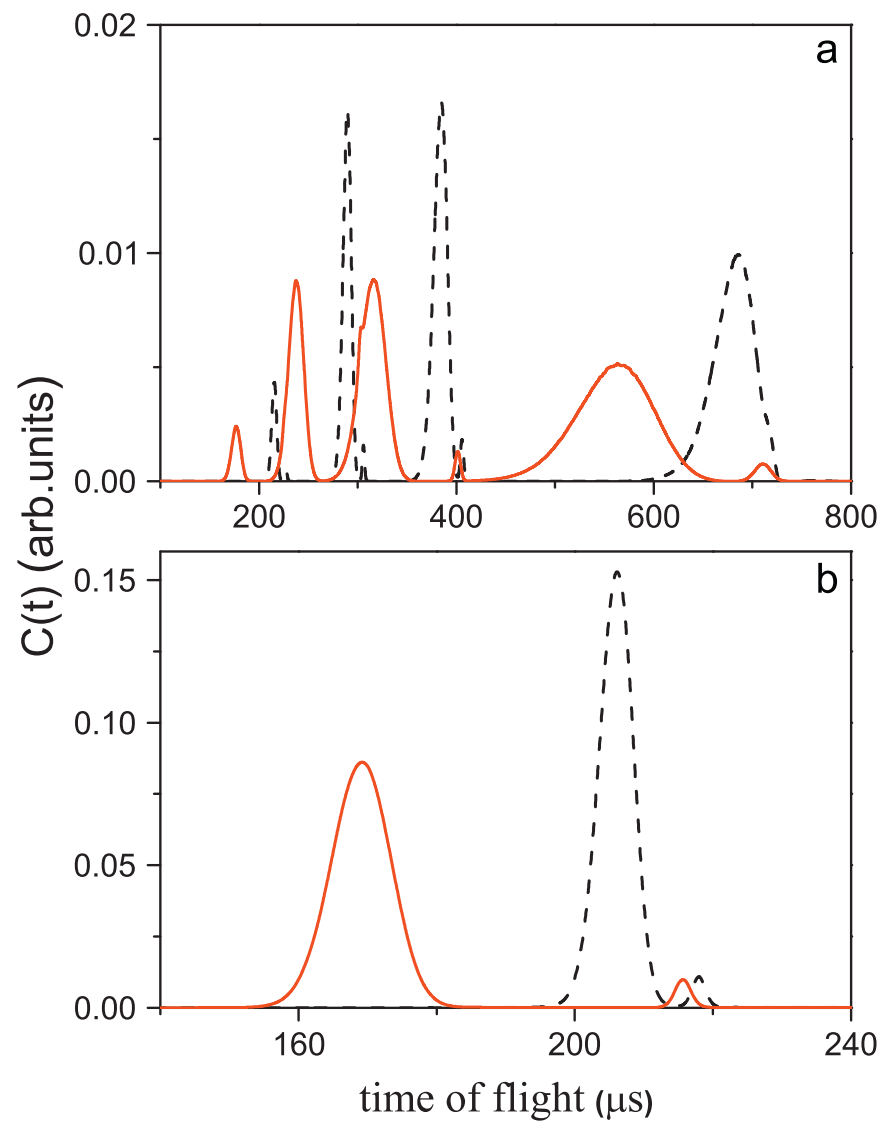

Fig. 10. Simulated tof spectra of $\mathrm{H}_{2} \mathrm{O}$ with the foil cycling technique for $L_{0}=25 \mathrm{~m}$, at $20^{\circ}$ (dashed line) and $40^{\circ}$ (solid line) for (a) ${ }^{238} \mathrm{U}$ and (b) ${ }^{139} \mathrm{La}$ analyzers.

$y$ and proportional to $1 / q$ and $1 / q^{2}$ (see Eq. (6)). As already stressed before, the higher the wave vector and energy transfers achieved in the scattering process, the lower the effect of the FSE. This can be appreciated in Fig. 11 where the proton Compton 


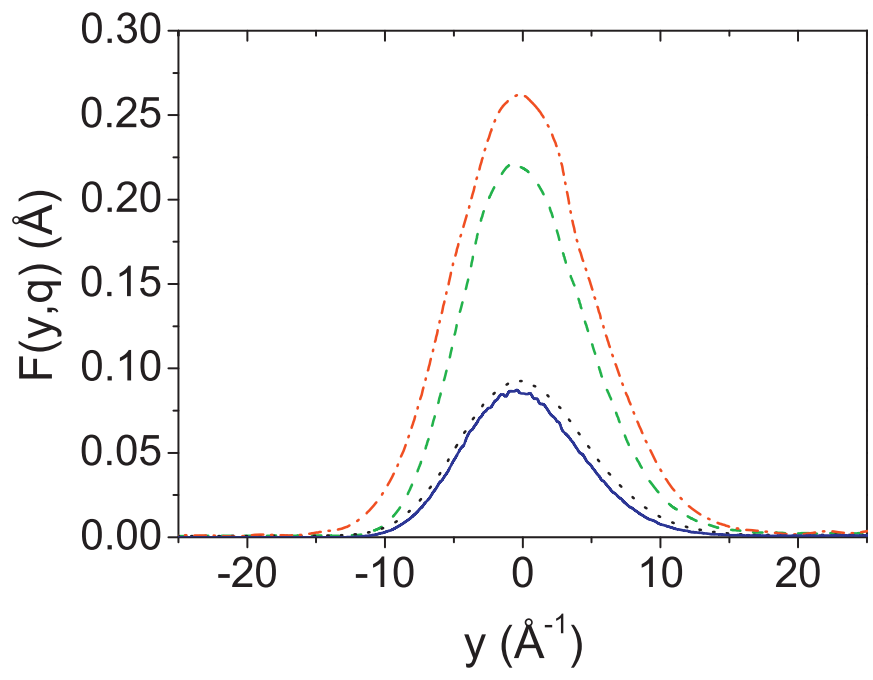

Fig. 11. Proton Compton profiles $F(y, q)$ obtained from the simulated time of flight spectra on a pure $\mathrm{H}$ sample, for $L_{0}=25 \mathrm{~m}$ and $2 \vartheta=30^{\circ}$ at final neutron energies of $6.67 \mathrm{eV}$ (continuous line), $20.9 \mathrm{eV}$ (dashed line), $36.6 \mathrm{eV}$ (dotted line) and $66.0 \mathrm{eV}$ (dot-dashed line).

profiles $F(y, q)$ obtained from the simulated time of flight spectra at the different final neutron energies selected by the ${ }^{238} \mathrm{U}$ analyzer are compared for fixed values of $L_{0}=25 \mathrm{~m}$ and $29=30^{\circ}$. The continuous line is from the $6.671 \mathrm{eV}$ final neutron energy that corresponds to the lowest $q$ and $\hbar \omega$ values accessed in the measurement, while the dotted and dot-dashed profiles are from the higher resonances. It can be noticed that the profile from the lowest final neutron energy is shifted and distorted, while the other ones are almost symmetric and centered at $y=0$, within the uncertainties of the simulation. This means that with good degree of accuracy the FSE are negligible for the higher final neutron energies, so that the Compton profiles attained their asymptotic form. Thus a weighted sum procedure can be applied to sum up the experimental Compton profiles at the different final neutron energies, after proper normalization. Clearly, the same applies for the higher angles, as both $q$ and $\hbar \omega$ increase. In this way the normalized Compton profiles recorded at energies above the $6.671 \mathrm{eV}$ of the ${ }^{238} \mathrm{U}$ analyzer can be properly summed together (in the $y$ space) for all the scattering angles, such that ${ }^{1} \mathrm{H}$ and ${ }^{16} \mathrm{O}$ peaks are well separated. In this way one obtains a single "sum recoil peak" to be analyzed, the corresponding resolution functions being the weighted sum of the resolution functions of the single $F(y)$ that are summed (Convolution Approximation, the weighting factor being the statistical error of data points). The resulting "sum recoil peak" is then characterized by a higher statistical quality, thus allowing for a more precise line shape analysis.

\section{Discussion and conclusions}

From the first set of simulations performed without considering the FSE, it can be seen that in the case of $L_{0}=11 \mathrm{~m}$ and the typical angular range of the VESUVIO spectrometer, the optimized 29 interval relatively to peak separation is between $25^{\circ}$ and $35^{\circ}$ for ${ }^{238} \mathrm{U}$, while for ${ }^{139} \mathrm{La}$ the whole typical angular range for DINS measurements can be exploited. If a longer flight path is considered (second set of simulations) an enhancement of the resolution can be obtained, due to the lowering of the contribution depending on $L_{1} / L_{0}[50]$ (as $L_{1}$ is fixed).

As expected on theoretical grounds, the simulations including the FSE show both a recoil peak shift and asymmetries in the line shape [51] at low scattering angles, where the lowest $\hbar \omega$ and $q$ are achieved. On the contrary, at low times of flight (i.e. at high energy and wave vector transfers) the FSE are negligible. Indeed, the higher the $q$ and $\hbar \omega$ accessed, the lower the FSE contribution (see Eq. (6)). Comparing simulations with and without FSE at $2 \vartheta=30^{\circ}$, it can be noted that FSE do not introduce substantial shifts or broadening of the peaks in all of the considered cases, with the possible exception of the peak corresponding to the $6.671 \mathrm{eV}$ resonance. Notice that for resonances at $20.8 \mathrm{eV}, 36.6 \mathrm{eV}$ and $66.0 \mathrm{eV}\left({ }^{238} \mathrm{U}\right)$ the IA approximation holds with a very good degree of accuracy, as the FSE contribution cannot even be appreciated. In the case of ${ }^{139} \mathrm{La}$ ( $72 \mathrm{eV}$ final neutron energy) the FSE contribution is practically negligible in almost all the cases undertaken in the simulations.

The use of the FCT guarantees that the energy transfer function of the analyzer-cycling foil system has a narrower FWHM as compared to the standard RD configuration, as shown in Figs. 9 and 10, while the effect on the data has been already shown in Refs. [34,35]. This is well shown in Fig. 12, where two simulated tof spectra of $\mathrm{H}_{2} \mathrm{O}$ with the FCT or the RD configuration (for a fixed scattering angle) are shown.

It can be concluded that in the case of the uranium analyzer, there is an angular range where the recoil peaks at different final neutron energies are all well separated and FSE are found to be negligible. As the data analysis is generally performed in the West scaling variable space, it could be possible to perform a weighted sum of the normalized recoil peaks (in $y$ space) corresponding to

a

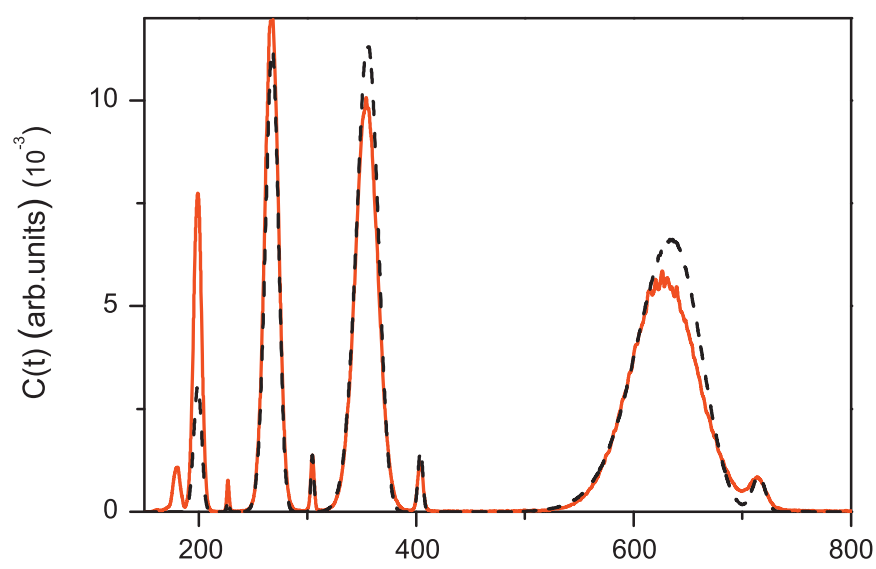

b

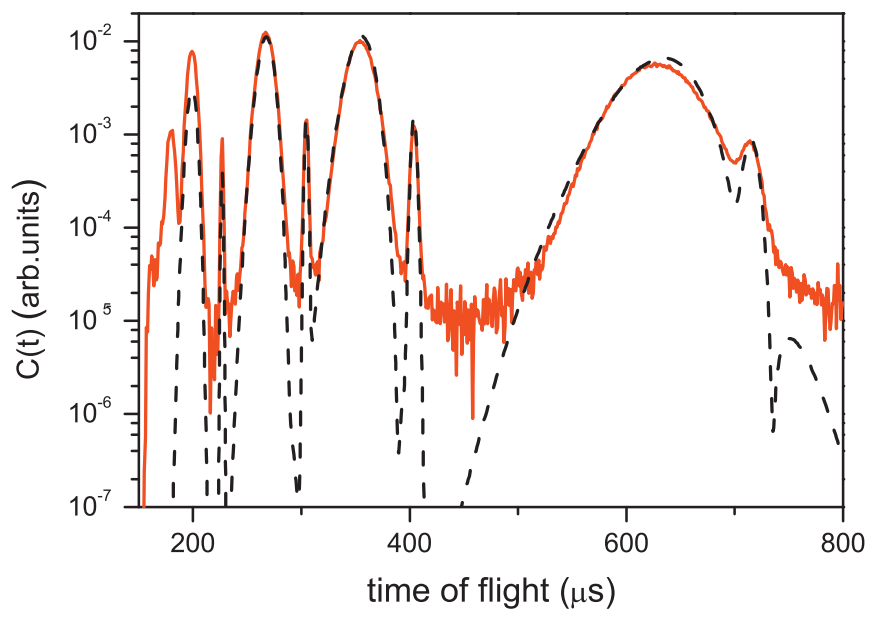

Fig. 12. Two simulated tof spectra of $\mathrm{H}_{2} \mathrm{O}$ with $L_{0}=25 \mathrm{~m}$, at $30^{\circ}$, with $\mathrm{FCT}$ (dashed line) and with the RD (solid line) configuration. Panels (a) and (b) show the tof spectra in linear and logarithmic scale, respectively. 
the resonances at 20.8, 36.6 and $66.0 \mathrm{eV}$ at all angles, $2 \vartheta$ between $25^{\circ}$ and $35^{\circ}$. In the $y$ space the resolution function of the "sum recoil peak" is given, in the convolution approximation (that holds with an excellent degree of accuracy) by the weighted sum of the resolutions of the summed detectors, thus providing a smooth recoil peak with a good statistical quality, the weighting factor being the statistical errors on each data point. Indeed, considering that detector spacing in the VESUVIO spectrometer is about $1-2^{\circ}$ and that an angular range of about $10^{\circ}$ is exploitable, a total of 25-30 spectra could be eventually summed together. The peaks corresponding to the $6.67 \mathrm{eV}$ uranium resonance can be treated separately as it happens for the actual experiments on VESUVIO $[3,4]$.

In the case of Lanthanum it is possible to exploit a more extended scattering angular range as compared to the case of uranium. Therefore, although only one recoil peak per scattering angle is available in this case, a total of about 20 spectra could be summed to obtain the "sum recoil peak", considering that detectors may be placed in the full (typical) angular range $\left(20^{\circ}-60^{\circ}\right)$ with a spacing of $1.5-2^{\circ}$.

In conclusion, a feasibility study has been presented to show that the RD configuration would allow to design an instrument capable of accessing a wider $q$ and $\hbar \omega$ range, as compared to the one presently investigated in DINS measurements from hydrogen containing samples. Despite its explorative nature, this work may stimulate further investigations, for example considering other analyzer materials and/or geometrical setup for a better separation of the peaks.

\section{Acknowledgments}

One of the authors (AP) acknowledges the CNR-CNISM joint research program.

\section{References}

[1] G.I. Watson, J. Phys.: Condens. Matter 8 (1996) 5955

[2] C. Andreani, D. Colognesi, J. Mayers, G.F. Reiter, R. Senesi, Adv. Phys. 54 (2006) 377.

[3] A. Pietropaolo, C. Andreani, R. Senesi, A. Botti, M.A. Ricci, F. Bruni, Phys. Rev. Lett. 100 (2008) 127801.

[4] C. Pantalei, A. Pietropaolo, R. Senesi, S. Imberti, C. Andreani, J. Mayers, C. Burnham, G.F. Reiter, Phys. Rev. Lett. 100 (2008) 177801.

[5] M. Ceriotti, G. Miceli, A. Pietropaolo, D. Colognesi, A. Nale, M. Catti, M. Bernasconi, M. Parrinello, Phys. Rev. B 82 (2010) 174306

[6] A. Pietropaolo, D. Fernandez-Cañoto, E. Perelli-Cippo, S. Dirè, P. Prosposito, Phys. Rev. B 77 (2008) 014202.

[7] G.F. Reiter, J. Mayers, P. Platzman, Phys. Rev. Lett. 89 (2002) 135505

[8] J. Mayers, Phys. Rev. Lett. 84 (2000) 314.

[9] R. Senesi, C. Andreani, D. Colognesi, A. Cunsolo, M. Nardone, Phys. Rev. Lett. 86 (2001) 4584

[10] C.G. Windsor, Pulsed Neutron Scattering, Taylor \& Francis Ltd, London, 1981.

[11] C. Andreani, A. D’Angelo, G. Gorini, S. Imberti, A. Pietropaolo, N. Rhodes, E. Schooneveld, R. Senesi, M. Tardocchi, Appl. Phys. A 78 (2003) 903.

[12] R.N. Sinclair, M.C. Moxon, J.M. Carpenter, Bull. Am. Phys. Soc. 22 (1977) 101; D.R. Allen, E.W.J. Mitchell, R.N. Sinclair, J. Phys. E: Sci. Instr. 13 (1983) 639.

[13] H. Rauh, N. Watanabe, Nucl. Instr. and Meth. 228 (1984) 147; N. Watanabe, in: Proceedings of the 1984 Workshop on High Energy Excitations in Condensed Matter, Los Alamos, 13-15 February, 1984 LA-10227-C, vol. 1.

J.M. Carpenter, N. Watanabe, S. Ikeda, Y. Masuda, S. Sato, H. Rauh, Physica B 120 (1983) 126.
[14] C.D. Bowman, R.G. Johnson, in: J. Faber Jr. (Ed.), AIP Conference Proceedings of 89 Neutron Scattering, 1981; R.G. Johnson, C.D. Bowman, Phys. Rev. Lett. 49 (1982) 797

[15] L. Cser, N. Kroó, P. Pacher, V.G. Simkin, E.V. Vasilieva, Nucl. Instr. and Meth. 179 (1981) 515

[16] J.M. Carpenter, N. Watanabe, S. Ikeda, Y. Masuda, S. Sato, H. Rauh, Physica B 120 (1983) 126

[17] N. Watanabe, in: Proceedings of the 1984 Workshop on High Energy Excitations in Condensed Matter, Los Alamos, 13-15 February, 1984, LA-10227-C, vol. 1.

[18] M.P. Paoli, et al., in: ICANS VIII: Proceedings of the Eighth Meeting of the International Collaboration on Advanced Neutron Sources, RAL-85-110, Rutherford Appleton Laboratory, vol. 577, 1985.

[19] R.K. Crowford, in: ICANS IX: Proceedings of the Ninth Meeting of the International Collaboration on Advanced Neutron Sources, ISBN 3-907998-01-4, Swiss Institute for Nuclear Research, Villigen, Switzerland, 1986, vol. 397.

[20] R.G. Johnson, C.D. Bowman, Phys. Rev. Lett. 49 (1982) 797.

[21] R.G. Johnson, Nucl. Instr. and Meth. A 163 (1985) 427.

[22] H. Rauh, S. Ikeda, N. Watanabe, Nucl. Instr. and Meth. 224 (1984) 469.

[23] C. Andreani, A. Pietropaolo, R. Senesi, G. Gorini, M. Tardocchi, A. Bracco, N.J. Rhodes, E.M. Schooneveld, Nucl. Instr. and Meth. A 481 (2002) 509.

[24] A. Pietropaolo, C. Andreani, A. D'Angelo, G. Gorini, S. Imberti, M. Tardocchi, N. Rhodes, E.M. Schooneveld, R. Senesi, in: Proceedings of the Eleventh International Symposium on Capture Gamma-Ray Spectroscopy and Related Topics, World Scientific, Singapore, vol. 555, 2003.

[25] M. Tardocchi, A. Pietropaolo, C. Andreani, A. Bracco, A. D'Angelo, G Gorini, S. Imberti, R. Senesi, N.J. Rhodes, E.M. Schooneveld, Nucl. Instr. and Meth. A 526 (2004) 477

[26] M. Tardocchi, G. Gorini, A. Pietropaolo, C. Andreani, R. Senesi, N. Rhodes, E.M. Schooneveld, Rev. Sci. Instr. 75 (2004) 4880.

[27] A. Pietropaolo, C. Andreani, A. Filabozzi, G. Gorini, N. Rhodes, R. Senesi, E. Schooneveld, M. Tardocchi, J. Instr. 1 (2006) P04001. doi:10.1088/ 1748-0221/1/04/P04001.

[28] E. Perelli-Cippo, G. Gorini, M. Tardocchi, C. Andreani, A. Pietropaolo, R. Senesi, N.J. Rhodes, E.M. Schoonveld, Appl. Phys. A 83 (2006) 453. doi:10.1007/ s00339-006-3570-8.

[29] R. Senesi, C. Andreani, Z. Bowden, D. Colognesi, E. Degiorgi, A.L. Fielding, J. Mayers, M. Nardone, J. Norris, M. Praitano, N.J. Rhodes, W.G. Stirling, J. Tomkinson, C. Uden, Physica B 276-278 (2000) S189.

[30] A. Pietropaolo, Research and Development of Gamma Detectors for Neutron Scattering at Electron Volt Energies on the VESUVIO Spectrometer, Ph.D. Dissertation, Roma: Universitá degli Studi di Roma Tor Vergata, 2004.

[31] V.F. Sears, Phys. Rev. B 30 (1984) 44.

[32] R.N. Silver, Phys. Rev. B 37 (1988) 3794.

[33] R.N. Silver, Phys. Rev. B 39 (1989) 4022.

[34] E.M. Schooneveld, A. Pietropaolo, C. Andreani, G. Gorini, J. Mayers, E. PerelliCippo, N.J. Rhodes, R. Senesi, M. Tardocchi, Rev. Sci. Instr. 77 (2006) 095103.

[35] A. Pietropaolo, A. Filabozzi, C. Andreani, E. Pace, R. Senesi, Nucl. Instr. and Meth. A 570 (2007) 498.

[36] R. Senesi, D. Colognesi, A. Pietropaolo, T. Abdul-Redah, Phys. Rev. B 72 (2005) 054119.

[37] P.C. Hoemberg, P.M. Platzman, Phys. Rev. 152 (1966) 198

[38] G.B. West, Phys. Rev. 18 (1975) 263 E. Pace, G. Salmé, G. West, Phys. Lett. B 273 (1991) 205

[39] S.R. Deans, The Radon Transform and Some of Its Applications, John Wiley \& Sons, New York, 1983.

[40] A.S. Rinat, Phys. Rev. B 36 (1987) 5171.

[41] H.A. Gersch, L.J. Rodriguez, P.N. Smith, Phys. Rev. A 5 (1972) 1547; H.A. Gersch, L.J. Rodriguez, Phys. Rev. A 8 (1973) 905

[42] H.R. Glyde, Phys. Rev. B 50 (1994) 6726.

[43] A. Rahman, K.S. Singwi, A. Sjölander, Phys. Rev. 126 (1962) 986.

[44] A. Pietropaolo, M. Tardocchi, E.M. Schooneveld, R. Senesi, Nucl. Instr. and Meth. A 568 (2006) 826.

[45] A. Pietropaolo, E. Perelli Cippo, G. Gorini, M. Tardocchi, E.M. Schooneveld, C. Andreani, R. Senesi, Nucl. Instr. and Meth. A 608 (2009) 121.

[46] J. Mayers, A.L. Fielding, R. Senesi, Nucl. Instr. and Meth. A 481 (2002) 454.

[47] R.J. Newport, M.P. Paoli, V.T. Pugh, R.N. Sinclair, A.D. Taylor, W.G. Williams, in: ICANS VIII: Proceedings of the Eighth Meeting of the International Collaboration on Advanced Neutron Sources, RAL-85-110 Rutherford Appleton Laboratory, 1985, p. 562.

[48] G.F. Reiter, J.C. Li, J. Mayers, T. Abdul-Redah, P. Platzman, Bras. J. Phys. 34 (2004) 142.

[49] A.L. Fielding, J. Mayers, Nucl. Instr. and Meth. A 480 (2002) 680.

[50] S. Imberti, C. Andreani, V. Garbuio, G. Gorini, A. Pietropaolo, R. Senesi, M. Tardocchi, Nucl. Instr. and Meth. A 552 (2005) 463.

[51] C. Andreani, A. Filabozzi, E. Pace, J. Mayers, Phys. Rev. B 54 (1996) 6255 